\title{
Modelling the fate and transport of colloidal particles in association with BPA in river water
}

\begin{abstract}
A simplified modelling approach for illustrating the fate of emerging pollutants can improve risk assessment of these chemicals. Once released into aquatic environments, these pollutants will interact with various substances including suspended particles, colloidal or nano particles, which will greatly influence their distribution and ultimate fate. Understanding these interactions in aquatic environments continues to be an important issue because of their possible risk. In this study, bisphenol A (BPA) in the water column of Bentong River, Malaysia, was investigated in both its soluble and colloidal phase. A spatially explicit hydrological model was established to illustrate the associated dispersion processes of colloidal-bound BPA. Modelling results demonstrated the significance of spatial detail in predicting hot spots or peak concentrations of colloidal-bound BPA in the sediment and water columns as well. The magnitude and setting of such spots were system based and depended mainly on flow conditions. The results highlighted the effects of colloidal particles' concentration and density on BPA's removal from the water column. It also demonstrated the tendency of colloidal particles to aggregate and the impact all these processes had on BPA's transport potential and fate in a river water. All scenarios showed that after 7.5-10 km mark BPA's concentration started to reach a steady state with very low concentrations which indicated that a downstream transport of colloidal-bound BPA was less likely due to minute BPA levels.
\end{abstract}

Keyword: BPA; Colloids; Water quality models; Aggregation; Adsorption; Transport 